\title{
A Declaration of the Dependence of Cyberspace
}

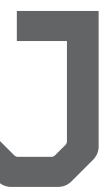

OHN PERRY BARLOW, the famed founder of the Electronic Frontier Foundation, a digital-rights advocacy group, passed away on Feb. 6,2018 . In 1996, Barlow published "A Declaration of the Independence of Cyberspace." It offered a rebuttal to Internet governance by national governments, opening with "Governments of the Industrial World, you weary giants of flesh and steel, I come from Cyberspace, the new home of Mind. On behalf of the future, I ask you of the past to leave us alone."

It is hard to believe that such a naïve view of cyberspace was taken seriously just about 20 years ago, that people really believed simplistic statements such as "We believe that from ethics, enlightened self-interest, and the commonweal, our governance will emerge." But we must remember that 20 years ago the Internet was indeed some kind of a New World, seemingly outside the shackling legacy of traditional governance. That was also before the Internet and the World Wide Web became dominated by giant corporations, and before Tim Berners-Lee, the recipient of the 2016 ACM Turing Award for inventing the World Wide Web, declared in 2017 that "The system is failing:"

What we have also learned in the past 20 years that while cyberspace may be indeed "the new home of Mind," it is inextricably connected to the physical world. Indeed, the economic impact of the Web has been and continues to be profound. Newspapers are struggling to survive because advertising income, which has been their main source of revenue, has migrated to the Web, with Google and Facebook as the main beneficiaries. While e-commerce escalates, traditional retail outlets are shuttering down daily, suffering from "retail apocalypse." And while cyberattacks are now a daily occurrence, there are growing fears of a possible cyberattack that will knock out U.S. power grids. ${ }^{b}$ What happens in cyberspace does not stay in cyberspace!

To my mind, however, nothing epitomizes the hubris of the technoutopianists more than the idea of reinventing money. In October 2008, the mysterious Satoshi Nakamoto posted a white paper $^{\mathrm{c}}$ on the new domain of bitcoin.org, in which he asserted, "What is needed is an electronic payment system based on cryptographic proof instead of trust." Bitcoin is based on a P2P network where transactions are cryptographically verified and recorded in a public distributed ledger based on blockchain, a distributed-consensus protocol. We now seem to be in the midst of a bitcoin mania, with the value of bitcoins gyrating wildly, making double-digit moves in a single week. There is also significant evidence ${ }^{\mathrm{d}}$ that the bitcoin-exchange markets are being manipulated. But bitcoin has only been the first of dozens of cryptocurrencies, and initial coin offerings, which raise funds for issuing new cryptocurrencies, and are growing in popularity.

But even if bitcoin solved (quite imperfectly) the verifiability and distributed-trust issues, the idea of apolitical money is a dangerous fantasy. Verifiability and trust are only two requirements from a currency. Other requirements, which are intimately related, are value and supply. Central banks used to strive for a stable currency value. More

\footnotetext{
a https://goo.gl/PEMP55

b https://goo.gl/esziCL

c https://goo.gl/55N41V

d https://goo.gl/dP1GTz
}

recently they have come to realize that a slightly depreciating currency value (about 2\% per year) is better for economic growth. To achieve this, central bankers use a variety of sophisticated monetary tools to manage the money supply, taking into account a large number of economic indicators. This is an enormously complicated task challenging the best economic minds. In contrast, the supply of cryptocurrencies is a priori limited, and its gyrating value is determined by trading decisions made by "investors." So the idea that apolitical cryptocurrencies will replace political money is a delusion.

Just like other speculative bubbles, the cryptocurrency bubble will also blow up at some point, though the timing is quite unpredictable. But the risk is not only to gullible speculators. As time goes on, cryptocurrencies get more enmeshed in our economic system and the risk of financial contagion grows. Financial contagion refers to the spread of market disturbances, typically on the downside, between different economic institutions and between different countries. The cryptocurrency bubble is, in my opinion, a growing systemic financial risk (and there are also the issues of susceptibility to cyberattacks and voracious energy appetite).

Just as you cannot separate the mind and the body, you cannot separate cyberspace and physical space. It is time to accept this dependence and act accordingly.

Follow me on Facebook, Google+, and Twitter.

Moshe Y. Vardi (vardi@cs.rice.edu) is the Karen Ostrum George Distinguished Service Professor in Computational Engineering and Director of the Ken Kennedy Institute for Information Technology at Rice University, Houston, TX, USA. $\mathrm{He}$ is the former Editor-in-Chief of Communications.

Copyright held by author 